\title{
LE DEVQHB
}

Le Devoir, Montréal, Édition du 17 juin 1998.

\section{Déclaration de Calgary - L'expérience récente comme seul passé}

Le document concocté en septembre 1997 synthétise l'affrontement de deux logiques égalitaires: l'égalité des personnes, des provinces et des chances s'oppose à 1'égalité des peuples et des conditions

Alain G. Gagnon, politologue, UQÀM

9. Courriel : gagnon.alain@uqam.ca

Avec l'autorisation formelle de l'auteur de diffuser toutes ses publications dans Les Classiques des sciences sociales, le 16 mars 2006.

Version abrégée d'un mémoire présenté devant les membres de la Commission parlementaire sur les Institutions, Assemblée nationale, gouvernement du Québec, le 10 juin 1998, à Québec.
Depuis le référendum d'octobre 1995 où l'option souverainiste a failli l'emporter, le gouvernement fédéral s'évertue, contre toute attente, à rendre plus difficile que jamais toute réforme constitutionnelle. Les ministres fédéraux se sont plu à parler de plan $\mathrm{A}$ et de plan $\mathrm{B}$ pour contraindre les Québécois à accepter le statu quo maintenant qualifié d'évolutif et à s'en réjouir. Ces deux angles d'approche - le plan A et le plan $B$ - font partie d'une seule et même stratégie: l'imposition de l'ordre constitutionnel canadien de 1982. Cette imposition se fait soit en ignorant tout simplement 
les revendications du Québec dans des champs aussi diversifiés que le sont, entre autres, la santé, la culture et l'éducation, soit par la voie de l'intervention du gouvernement fédéral dans les champs de compétence exclusive aux provinces (pouvoir fédéral de dépenser, pouvoir déclaratoire et pouvoir de désaveu), soit encore par l'intimidation, c'est-à-dire en faisant appel à la contrainte (le renvoi devant la Cour suprême et, même, la menace à peine voilée de faire appel à l'armée en suggérant que les initiatives prises par le gouvernement du Québec manquent de transparence).

C'est dans ce contexte qu'il faut situer la Déclaration de Calgary. En quoi cette déclaration peutelle permettre de répondre aux revendications du Québec et en quoi est-elle en mesure de réconcilier le Québec avec l'ordre constitutionnel qui lui a été imposé en 1982?

Soulignons que le gouvernement fédéral a jusqu'ici refusé de donner son appui officiel à la Déclaration de Calgary. Soulignons aussi que la déclaration a fait l'objet de nombreuses réserves dans plusieurs provinces. A ce jour, les principaux intervenants ayant fait des démarches lors des audiences publiques tenues à l'extérieur du Québec y sont allés de propos vagues. Il est loin d'être acquis que les Canadiens seront conviés à se prononcer lors d'un référendum sur les mérites de la déclaration. Le danger d'un rejet et d'une polarisation accrue quant aux tenants et aboutissants de la déclaration est trop sérieux pour que le gouvernement fédéral s'engage naïvement dans une pareille démarche.

Le principal objectif poursuivi par les provinces et, derrière celles-ci, le gouvernement fédéral, se limite simplement à dire que la reprise des négociations constitutionnelles entre le Québec et ses partenaires canadiens devra se faire dans un cadre de discussion prédéterminé qui ne tient aucunement compte de la notion même de peuple québécois.

Les auteurs de la déclaration rappellent leur volonté commune de faire des propositions pouvant permettre aux Québécois d'adhérer à la fédération canadienne. Les auteurs se proposent de défendre concurremment l'égalité des provinces, l'égalité des personnes et l'égalité des chances. En outre, 
on reprend la notion d'asymétrie qui était au coeur du rapport de la commission Pepin-Robarts en 1979. Sans représenter une percée significative au chapitre du partage des pouvoirs, l'utilisation de cette avenue pourrait toutefois apporter certains accommodements susceptibles d'intéresser les représentants des partis politiques à Québec. Il faut signaler toutefois la mauvaise perception que les Canadiens ont du concept d'asymétrie, qu'ils réduisent souvent à des privilèges. Les auteurs de la déclaration ont cherché à contrer cette critique en indiquant que toutes les provinces pourraient s'en prévaloir.

On fait référence en passant dans la déclaration que le Québec se caractérise par son caractère unique et on prend soin de limiter ses traits distinctifs à la majorité francophone, la culture et la tradition civiliste. Il est aussi précisé que l'Assemblée nationale («législative», selon le terme employé dans la déclaration) pourra en faire la promotion uniquement au sein du Canada.

Cela suggère donc que l'Assemblée nationale pourra veiller à l'affirmation du caractère unique de la société québécoise dans la mesure où elle respectera les règles du jeu que la fédération canadienne aura cru bon de lui imposer. Par ailleurs, les auteurs de la déclaration n'ont pas cru utile de proposer la constitutionnalisation de la notion de caractère unique de la société québécoise, la rendant par le fait même sans effet.

L'ouverture faite à l'expression des trois formes d'égalité auxquelles je viens de me référer (provinces, personnes, chances), sans d'ailleurs qu'un ordre de priorité n'ait même été établi entre celles-ci, passe sous silence la logique égalitaire qui tend à dominer la scène politique québécoise depuis le début des années 1960. Je me réfère ici à l'égalité des conditions et à l'égalité des peuples.

La Déclaration de Calgary oppose à la logique égalitaire québécoise, qui se fonde sur l'égalité des conditions et des peuples, une logique concurrente, favorisant l'ordre constitutionnel de 1982, ordre constitutionnel qui a été répudié, on le sait, à l'unanimité des partis politiques représentés à l'Assemblée nationale en 1982. 
La dualité culturelle - élément définisseur s'il en est un - à l'origine de la fédération canadienne se dissipe au profit d'un projet d'intégration pancanadien et est astreinte, selon les termes utilisés dans la déclaration, au dynamisme des langues française et anglaise (article 4). Toute référence aux peuples fondateurs s'est éteinte. Toute référence à l'histoire a disparu, comme si le Canada n'avait de passé que son expérience récente.

La Déclaration de Calgary souscrit à la notion générale de diversité comme fondement de l'unité canadienne mais les auteurs prennent bien soin de ne pas préciser de quelle diversité il s'agit.

La notion de diversité utilisée par les auteurs de la déclaration correspond à celle dont nous avons hérité de Pierre Trudeau dès 1971 et qui a été enchâssée dans la Constitution de 1982 sans le consentement du Québec. Le sens donné à la notion de diversité s'inscrit dans le même sillon et tourne le dos au sens que souhaiterait lui donner Charles Taylor pour sortir le Canada de la présente crise constitutionnelle.
Quant à l'idée d'égalité à laquelle j'ai fait allusion plus tôt, il faut aussi faire le même constat. Il ne s'agit pas pour les auteurs de la Déclaration de Calgary de faire amende honorable en reconnaissant l'injustice de 1982, selon l'expression même de l'un des plus grands spécialistes sur le fédéralisme canadien qu'était feu Donald Smiley.

Il est révélateur que les notions d'égalité des peuples et d'égalité des conditions soient absentes de la Déclaration de Calgary. Cela ne présage rien de bon pour le Québec quant à la signature de l'entente-cadre sur l'union sociale présentement à l'étude. A cet égard, rappelons que la déclaration va jusqu'à réclamer la participation du gouvernement fédéral dans la prestation des programmes sociaux, champ de compétence provinciale s'il en est un. Cette position commune des représentants territoriaux et provinciaux laisse entrevoir une fois de plus que les provinces hors Québec et que le Québec évoluent carrément selon des logiques distinctes.

La solidarité canadienne ne pourra se construire sans tenir compte de la logique égalitaire qui domine le champ politique au Québec. Egalité des peuples et 
égalité des conditions doivent, dans une perspective québécoise, avoir voix au chapitre, soustendre même les relations de pouvoir au sein de l'espace politique canadien.

La Déclaration de Calgary se révèle un fourre-tout. On y traite du fonctionnement de la fédération, des relations de pouvoir, de l'asymétrie, de la diversité, de la tolérance, de l'égalité des provinces et des individus, de la présence des peuples autochtones, du caractère multiculturel canadien, du dynamisme des langues française et anglaise, et une fois tous ces éléments réitérés, on y inscrit les particularismes québécois. On y parle de tout sauf de la question de fond, question qui remet d'ailleurs en question les fondements mêmes de la fédération canadienne, soit le droit inaliénable des Québécois de faire sécession démocratiquement.

Pour essayer de rendre acceptable la Déclaration de Calgary auprès des administrations territoriales et provinciales, il a fallu inscrire dans le texte une foule d'éléments qui ne tiennent aucunement compte des attentes du Québec et, surtout, qui sont en porte-à- faux avec les revendications exprimées par l'ensemble de la classe politique québécoise depuis l'injustice de 1982.

Malgré cette volonté politique territoriale et provinciale d'en arriver à une position commune, la Déclaration de Calgary ne fera sans doute pas l'objet d'une vraie consultation populaire auprès de tous les Canadiens parce que, comme l'ont fait l'entente du lac Meech et le projet de Charlottetown, elle polariserait à nouveau les Canadiens et ferait ressortir le peu d'ouverture des Canadiens hors Québec à l'égard de la communauté nationale québécoise. Pareille consultation est trop risquée pour le gouvernement fédéral; on peut donc s'attendre à ce qu'une fois tenue la prochaine élection provinciale au Québec, la Déclaration de Calgary soit reléguée aux oubliettes.

La Déclaration de Calgary, on l'aura deviné, n'a rien pour répondre aux attentes des souverainistes au Québec. Ce n'est pas l'objectif poursuivi par ses initiateurs. Qui plus est, cette même déclaration n'a rien non plus à offrir à ceux qui croient toujours que le Canada peut se renouveler en tenant compte des priorités de la société québécoise. 
C'est comme si le Canada se construisait sans égard pour cet Etat-membre de la fédération.
C'est comme si le Québec avait déjà quitté la fédération canadienne. 